\title{
SURVEY OF RESEARCH IN MODELING CONVEYOR-BASED AUTOMATED MATERIAL HANDLING SYSTEMS IN WAFER FABS
}

\author{
Dima Nazzal \\ Ahmed El-Nashar \\ Department of Industrial Engineering and Management Systems \\ 4000 Central Florida Blvd., University of Central Florida \\ Orlando, FL 32816, U.S.A.
}

\begin{abstract}
Automated material handling systems (AMHS) play a central role in modern wafer fabrication facilities (fabs). Typically, AMHS used in wafer fabs are based on discrete vehicle-based overhead systems such as overhead hoisted vehicles. Conveyor-based continuous flow transport (CFT) implementations are starting to gain support with the expectations that CFT systems will be capable of handling high-volume manufacturing transport requirements. This paper discusses literature related to models of conveyor systems in semiconductor fabs. A comprehensive overview of simulation-based models is provided. We also identify and discuss specific research problems and needs in the design and control of closed-loop conveyors. It is concluded that new analytical and simulation models of conveyor systems need to be developed to understand the behavior of such systems and bridge the gap between theoretical research and industry problems.
\end{abstract}

\section{INTRODUCTION}

Semiconductor manufacturing plays a major role in the global economy. It is one of the most complex industries involving highly sophisticated manufacturing processes. The process starts with raw wafers, a thin disc made of silicon or gallium arsenide. Depending on the diameter of the wafer, up to a few hundreds of identical chips can be made on each wafer, through building up the electronic circuits layer by layer (Schoeming 1999). As fabrication technology becomes more advanced, the size of features decreases. More cost reduction can be achieved by decreasing feature size and increasing wafer diameter as it yields more chips per wafer. The International Technology Roadmap for Semiconductors (ITRS) (Singer 2005) identifies the critical research and development needs of the semiconductor manufacturers over the next decade. According to the ITRS, the next wafer size will be 450 $\mathrm{mm}$.
The wafer fabrication process consists of re-entrant flow where wafers may enter the same process such as lithography, thirty or more times (Kuhl and Laubisch 2004). Wafer fabs typically process wafers in lots of 25 . The amount of processing that the device endures increases as the circuit design becomes more complex, for example a $300 \mathrm{~mm}$ wafer travels approximately from 8 to 10 miles during the processing and typically visits 250 process tools to undergo several hundred individual processing steps (Agrawal and Heragu 2006). As a result of the complex nature of fabrication, high productivity levels are not easily achieved.

This paper discusses literature related to models of conveyor systems in semiconductor fabs. A comprehensive overview of simulation-based models is provided followed by a brief discussion of general analytical models of closed loop conveyors. We also identify and discuss specific research problems and needs in the design and control of closed-loop conveyors.

The paper is organized as follows: In Section 2, we present a brief discussion of the common layout of $300 \mathrm{~mm}$ wafer fabrication facilities, and typical AMHS technologies. Section 3 discusses next generation conveyor-based AMHS, and the current literature on this topic. Section 4 identifies and specifies research needs that fall under closed loop conveyor-based AMHS implementations. Finally, our conclusions are presented in Section 5.

\section{AUTOMATED MATERIAL HANDLING SYSTEM}

Functional area layouts are adopted in wafer fabrication facilities, where similar groups of processing equipment are located together in a single area. Functional areas are further divided into groups of equipment called bays. The transportation system is composed of both interbay and intrabay transport equipment (Gartland 1999). 


\section{Nazzal and El-Nashar}

$300 \mathrm{~mm}$ fabs were designed to provide more flexibility and to eliminate bay pitch dependency on the stocker location. The stockers are separated from the bays and are located in a central tall corridor (Figure 1) which serves as the main corridor in the fab, in which the interbay AMHS is installed.

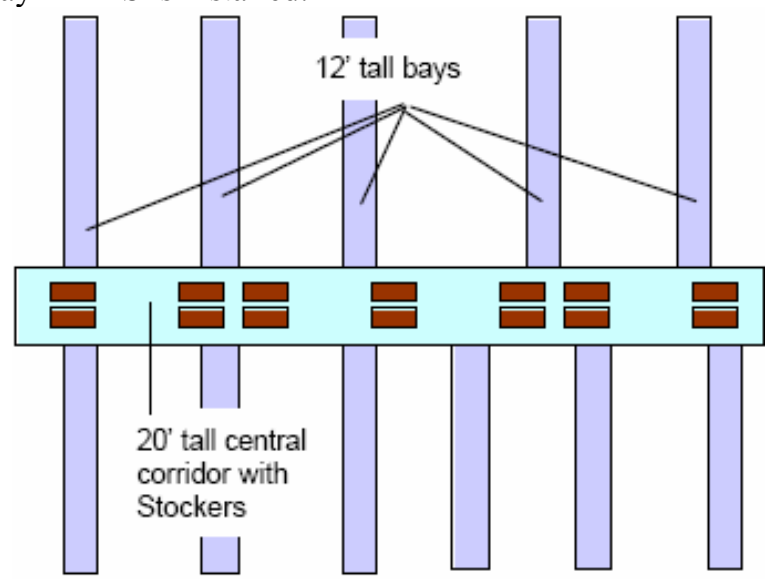

Figure 1: New layout design of the $300 \mathrm{~mm}$ fab (B. Shon).

In $300 \mathrm{~mm}$ wafer fabs, wafers are transported in a sealed carriers called Front Opening Unified Pod (FOUP) to keep them away from any source of contamination. The FOUP carries 25 wafers and it weighs in the range from 8 to $10 \mathrm{Kg}$ including both the wafers and FOUP weight (Foster 2001). Because of the ergonomic limitation posed by the weight and volume of the $300 \mathrm{~mm}$ wafer carrier, $300 \mathrm{~mm}$ factories and beyond require automated carrier handling (Ferrell and Pratt 2002).

The size of $300 \mathrm{~mm}$ wafers, their value, the increased number and complexity of process steps, the increased cost of the facility and equipment itself, and the chipmaker's competitive pressure to improve their own manufacturing economics; placed demands on a fab's automated material handling system requirements that even most advanced $200 \mathrm{~mm}$ fabs are unable to fulfill (Bonora, Brain et al. 2001). The slightest drift or malfunction at any of the handling or storage steps can cause a process deviation, which can ruin an entire lot or multiple lots. Such a process deviation is a costly one, as a single $300 \mathrm{~mm}$ wafers lot is roughly worth $\$ 1$ million (Therrien 2002).

The typical $300 \mathrm{~mm}$ AMHS configuration has a central material handling spine (interbay system) and loops branching on both sides to serve production equipment (intrabay systems) (figure 1). There are two distinct operating scenarios: (1) the spine and the loops are decoupled using stockers, AMHS is dedicated to the spine or to one of the loops, and lots moving between loops (or bays) require three different moves and two stocker moves; or (2) the spine and the loops are integrated, and lots moving between two loops require only a single move.
The $300 \mathrm{~mm}$ fab layout is a three-level automation system (Figure 2) with 20 feet tall stockers that all are interconnected by monorails and linked with every tool (production equipment) in the factory via an overhead vehicles transport system (OHV) (Garrett 2001). Stockers placed at the end of the bays are used for lot storage and may serve as a bridge between the interbay and intrabay systems where handling between the two AMHS systems occurs (Shikalgar et al. 2002; Kuhl and Christopher 2004).

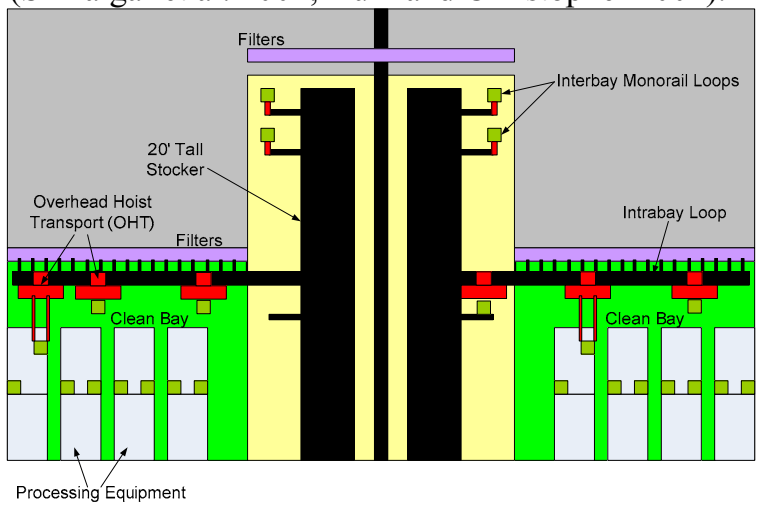

Figure 2: The $300 \mathrm{~mm}$ multilevel AMHS.

\subsection{Currently Implemented AMHS in wafer fabs}

Recently, the common AMHS used in wafer fabs are based on discrete vehicle-based overhead systems such as overhead hoisted vehicles (Figure 3), which replaced floor based systems such as the rail guided vehicles and automated guided vehicles because of its greater personal safety, smaller footprint, and better scalability (Roeder and Govind 2004).

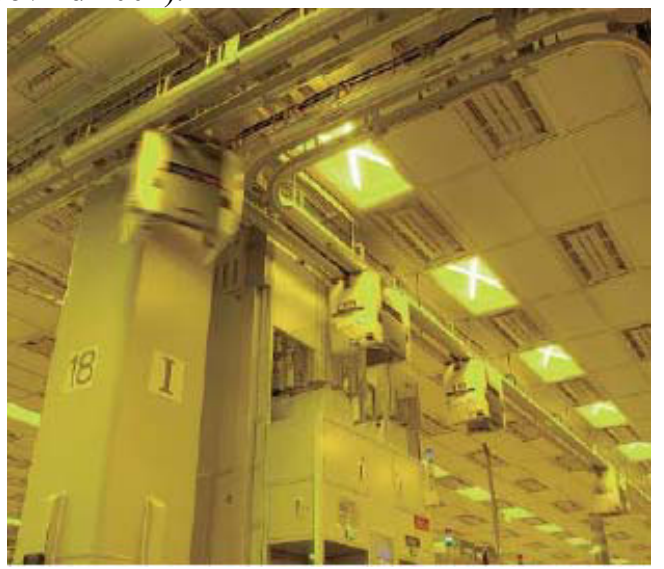

Figure 3: Overhead Hoisted Vehicles in $300 \mathrm{~mm}$ wafer fab.

The Overhead Monorail Vehicles (OMV) used by interbay or the Overhead Hoisted vehicles (OHV) intrabay are relatively efficient, and occupy little of the expensive clean room floor space as they travel on tracks suspended above the main fab floor, but advocates of conveyor systems (Arzt and Bulcke 2000; Paprotny, et al. 2000) claim that they are very expensive as the standard systems based 
on intrabay stockers and interbay monorails often exceed a cost of $\$ 30$ million, and the downtimes of such systems are frequent and costly.

\section{$2.2 \quad$ Next generation AMHS}

It is expected that wafer fabrication facilities in the near future will be characterized by the following (Feindel and Kaempf 2000):

- Higher traffic intensity caused by the predicted trend toward smaller batch or single wafer processing.

- Equipment scheduling demanding more predictability for when lots will be available for processing.

- Long distance and point-to-point transport between fab areas.

These characteristics necessitate the presence of automated material handling system with higher transport capacity, and shorter and more predictable delivery times. According to the 2005 ITRS, installing conveyor transport systems is a potential solution to meet the requirements of high throughput and low delivery times.

\section{CONTINUOUS FLOW TRANSPORT (CFT) APPROACH}

Although conveyor-based Continuous Flow Transport (CFT) implementations have been limited in the semiconductor industry, they are starting to gain support with the expectations that CFT systems will be capable of handling high-volume manufacturing transport requirements (ITRS, 2005).

The next generation of CFT AMHS addresses a direct process tool-to-process tool system that uses an overhead or floor-mounted conveyor system (having no vehicles) for material movement like that shown in Error! Reference source not found., this new system is coupled with local robotic handlers to transfer the carriers from the conveyor track to process tool load port (Arzt and Bulcke; Wright et al. 2002).

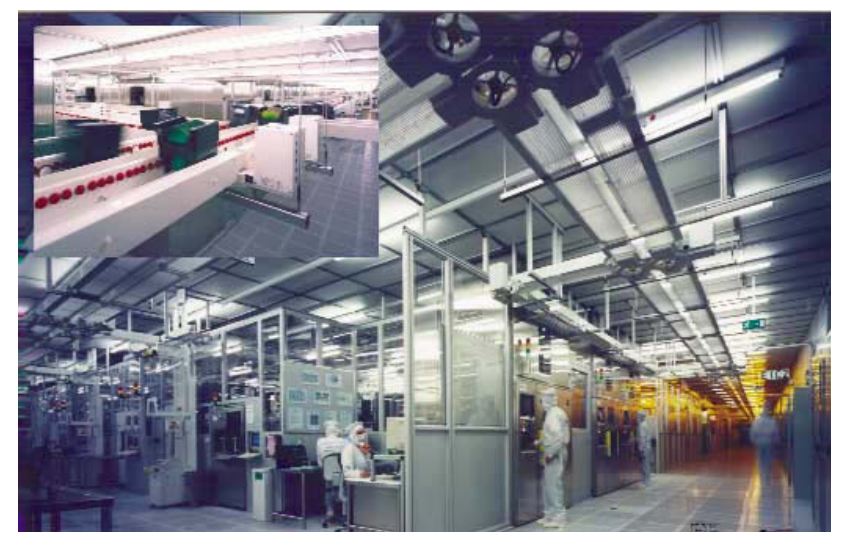

Figure 4: Continuous Flow Transport system (Heinrich and Pyke, 1999).

Some studies have been carried out to compare the performance of vehicle-based vs. conveyor-based material handling systems (Horn and Podgorski 1998; Paprotny, Shiau et al. 2000; Tausch and Hennessey 2002), the common approach used by these studies is to build a simulation model for a number of bays or for the full fab and compare the different proposed configurations by using a set of different performance measures.

Feindel and Kaempf (2000) discuss the advantages of CFT over traditional vehicle -based monorails and manual delivery systems. The paper argues that CFT systems are suited to satisfy the automation requirements of new fabs because they provide higher transport capacity, shorter and more predictable delivery times and lower cost-ofownership (CoO). They point out the capability of CFT to provide local buffers for process tools, which reduces the need for stockers. The article criticizes vehicle-based systems because their transport capacity is influenced by the number of alternate paths, the number and speed of the vehicles; and the control algorithms. Advantages of CFT were based on a simulation-based comparison of CFT and overhead hoist transport (OHT) systems across various levels of production, in which the CFT systems show faster delivery time with lower standard deviation.

Arzt and Bulcke (2000) present a simulation model of a wafer fab equipped with CFT system. The conveyor system was located on the sub floor of the wafer fab, where each conveyor loop was located exactly underneath the corresponding bay of the fab, the local intrabay buffers are divided into small sections or loops. Outside each bay, a lift allows the transportation down to the conveyor system. The paper discusses the benefits of process tools buffering provided by CFT systems as opposed to buffering provided by bay stockers

Paprotny et al. (2000) compare the performance of the OHT and CFT handling technologies through a simulation model of a low volume $300 \mathrm{~mm}$ wafer fab. The performance metric selected for comparison is the delivery time distribution. Their results indicate that OHT outperformed the CFT with delivery times being approximately 
half as large, which is attributed to the significantly slower transportation speed of the CFT. The standard deviation obtained from the OHT system is larger than that for the CFT model. Authors attribute the high variability of OHT performance to the uncertainty in the location and availability of vehicles, while in the case of CFT, the conveyor is always available, and a lot has to wait for free conveyor space to accommodate it. Authors conclude that CFT exhibits high throughput with more predictable delivery times. The authors also indicate that CFT is a lower cost alternative to OHT, which can be expensive, with cost projections for a $300 \mathrm{~mm}$ AMHS installation are in the range of $\$ 50 \mathrm{M}-\$ 100 \mathrm{M}$.

In their paper, Tausch and Hennessey (2002), compare the performance of a CFT system to an OHT system using simulation. The OHT system employs a segregated intrabay/interbay layout with Through Stocker (TS) lot transfer. The CFT system consists of conveyors with turntables. In the CFT system, lots use the conveyor as a means of delivery directly to a tool-loading module, which provides buffer storage for process tools and direct loading. The authors acknowledge the difficulty in performance metric selection because both systems operate differently. They select the following metrics; delivery time, transport time, throughout volume, throughput variability (measured by the coefficient of variability), and maximum throughput capability. Results exhibit faster delivery time for CFT, with lower variability and higher throughput. Authors attribute the advantages of CFT to its continuous availability for both transportation (and thus no time is spent waiting for transportation) and storage (that is closer to the destination process tool than a bay stocker).

Rust et al. (2002) compare three types of AMHS simulation models: OHT with Through Stocker (TS) transfer, OHT with Point-to-Point (PTP) transfer, and conveyor system with PTP transfer. In the conveyor model, if the finished lot is not immediately selected, it will be sent to circulate on the conveyor of the next station family bay. If the conveyor sections are at capacity, then one of the stockers serves as storage. Parameters measured included average moves per hour, WIP, transport times and delays waiting for transportation, process tool idle time waiting for lot to be delivered, process and cycle times by lot type, queue times by lot type and number of moves to and from stockers. Results exhibit similar product cycle time for all three models. The conveyor model exhibits the least amount of queue time. However, it also has the longest transportation component of overall cycle time. Conveyor model also exhibits short delays to lots waiting to access the conveyor caused by some occurrences of lots moving in a "train" (lots with minimum spacing on the conveyor). The authors attribute these occurrences to the slower speeds at turntables in the conveyor system and the short periods of blockage as lots are placed on or retrieved from conveyor, and recommend the use of merge/diverge sidings for high throughput tools or stockers to minimize track blockage. Furthermore, the average percentage of time that a tool is held idle waiting for a lot is the lowest for the conveyor model and this is because the logic of the conveyor system allows lots to be delivered to the bay of the next tool to process the lot, and circulate until the tool is ready, while in the OHT models, the lot is sent to the stocker until the tool is ready.

Horn and Podgorski (1998) favor CFT systems claiming that a conveyor based transport network will allow the same tool utilization as in a vehicle-based network at shorter cycle times. Authors repeatedly point out the indispensability of stockers in vehicle-based systems because they cannot provide buffer as conveyors do. This, the paper claims, prevents a demand pull operation with vehicles, which increases the overall WIP in the system, which has negative effects on the cycle time.

Heinrich and Pyke (2000) list the benefits of the conveyor based transport network at Infineon Technologies Fab in Dresden, Germany. They state that compared to the vehicle-based system, the conveyor implementation resulted in cleanroom space savings, elimination of transport batching, significant reductions in stocker requirements, and increase in tool utilization due to local buffering.

In 2003, International SEMATECH (ISMT) published an article on a transport network in one of its development facilities. The material handling network is an integration of an OHT system with a CBT system. The report claims that this system provides better throughput, more flexibility, and greater tool utilization over the conventional types of transport systems currently in use in the industry. The transport system combined one bidirectional conveyor system with an OHT for direct delivery to load ports using bi-directional vehicles and custom transfer points to and from the conveyor.

\subsection{The case for CFT systems}

Based on the above review, the conveyor-based systems have the following characteristics that enhance the performance of the system:

- Storage capabilities - when space is available on the conveyor, its continuous flow allows its use for FOUP storage, as opposed to sending the FOUP to a stocker, which is the case in OHT systems. This eliminates loading and retrieval from stockers, which might lead to cycle time improvement.

- High availability - in contrast to OHT systems, a lot does not need to wait for the conveyor once a move request has been issued. However, the lot must wait for a clear space on the conveyor. This eliminates the time spent waiting for vehi- 
cles, which could improve the cycle time. Standard deviation of delivery time could also decrease because waiting time for vehicles is less predictable than the time to access the conveyor is.

- Local buffering - the capability to serve as a local buffer is an advantage of CFT because the waiting FOUP stays close to the tool for immediate loading once the tools is ready to receive it. Since the FOUP stays close to the process tool, the time the tool stays idle waiting for the lot is reduced and the tool utilization could improve. In fact, Brain et al. (1999) and Feindel and Kaempf (2000) discuss the addition of short loops or cross buffers (Error! Reference source not found.) linked to the main track installed above the production tool that can serve as local zero-foot print FOUP buffers, which increases equipment effectiveness and reduces the need for large stockers at the end of each bay or larger process tool foot print.

- Simplified control: claim that local buffering simplifies scheduling because lots can be delivered to the buffer independent of the tool's status (Feindel and Kaempf, 2000).

- Low cost: according to Paprotny et al. (2000), CFT systems are lower in cost than OHT systems. Moreover, the lower number of required stockers has a direct impact on reducing the total system cost.

- Low space requirements: both OHT and CFT are installed overhead close to ceiling, and thus they are "zero-footprint". OHT systems require more stockers for local buffering, while CFT can utilize the conveyor segments for storage and the overhead loops for local buffering, resulting in less required storage space.

On the other hand, these studies favoring conveyorbased AMHS, fall short of pointing out some of the potential problems that can be observed with these CFT systems such as:

- The impact of AMHS components failures can be more severe in conveyor networks. A vehicle failure is recovered by taking it off the tracks and the other vehicles can be re-routed. A conveyor loop can be halted if a conveyor segment goes down.

- Schmidt and Jackman (2000) point out that if the loading and unloading schedules are not properly balanced with the maximum capacity of the conveyor and the stations, the number of recirculating loads can increase to the capacity of the conveyor, creating deadlocks.

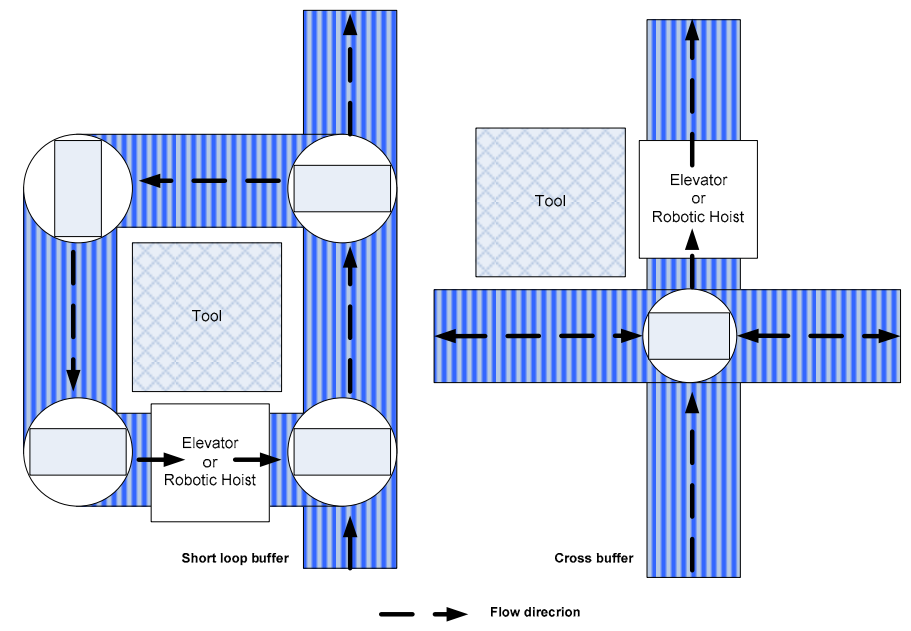

Figure 5: Plan over view of Zero foot print CFT buffer (Brain et. al 1999).

- Conveyors are categorized under synchronous AMHS, which implies that changing conveyor speed propagates throughout the network. Sometimes, it is desirable to speed up certain segments of the conveyor without affecting others.

- Flexibility of CFT network design is questionable. In OHT systems, vehicles are added and taken of the tracks to change the system's performance, such as the MHS throughput. CFT systems are less easily modified, increasing the speed, if technologically possible, could increase throughput but as was stated before, it might generate some undesirable changes in other areas in the network.

While there are some advantages for CFT over OHT and vice versa, one technology does not outperform the other in every setting. Each fab has its own requirements and characteristics that require different transport technologies. Decisions need to be based on the particular fab parameters and its bottom-line. Fair comparisons are those based on "good" designs, a good design is one based on sound methods and smart control rules. In one of the aforementioned studies, OHT model used a 25wafer lot, while CFT used a 50-wafer lot, this will affect the volume of traffic on both systems and thus degrade OHT by increasing the flow requirements on vehicles, while bringing advantage to the CFT systems by reducing the traffic on conveyors and thus lots have faster access to conveyors. Furthermore, the performance of both vehicle-based and conveyor-based systems is highly influenced by the operational policies for dispatching and routing, and well-founded conclusions cannot be made without a side-by side comparison between two "smart" implementations for each type of transport systems. 


\subsection{Modeling closed loop conveyors}

The ability of closed-loop conveyors to provide storage and transportation led to extensive development of performance analysis models. Conveyors can be studied with the use of simulation. Models of real systems are designed and experiments are performed with these models to gain an understanding of the behavior of these systems. However, the industry and research literature can benefit from analytical modeling tools that provide quick, generic tools to analyze and understand the behavior of conveyors.

Throughout the literature, analytical conveyor models have fallen into two categories: deterministic and stochastic models. Kwo (1959), Muth and White (1979), Muth (1972) and Muth (1975), Bastani (1988) studied the deterministic version of the problem and investigated feasibility conditions for operating simple closed-loop conveyors.

For the stochastic problem, Mayer (1960) develops a probabilistic model for a closed loop conveyor carrying discrete units away from $\mathrm{n}$ independent loading stations. Sonderman (1982) analyzes the effect of recirculation on the overall behavior of the system.

Coffman and Ngelenbe (1988) analyze conveyor queues in a flexible manufacturing system, they study the effect of the distance separating the input and output points of a workstation, where one or two robots unload and load parts from and to the conveyor. Schmidt and Jackman (2000) model a recirculating conveyor as an open network of queues. The system consists of one loading station, one unloading station, and two servers performing the same service on loads entering the system, and a loop conveyor divided into segments. Atmaca (2001) studies a model of a flexible manufacturing system consisting of workstations and a circular conveyor having a finite number of cells. The paper presents a method to analyze the conveyor system in which workstations have finite capacity queues to obtain main system time, mean number of work pieces, the probability a cell is occupied by a work piece and conveyor's throughput. Bozer and Hsieh (2004) extend the system studied by Atmaca (2001) to consider multiple loading and unloading stations and present an analytical model to approximate the expected waiting times experienced by loads that arrive at the loading stations.

\section{CFT RESEARCH PROBLEMS}

Although, conveyor-based implementations are envisioned to meet the requirements for future $450 \mathrm{~mm}$ fabs and high-volume manufacturing, not enough research has been done addressing design and control problems for these systems; all of the studies have been simulationbased, application-specific that cannot be generalized.
Moreover, there are significant gaps between existing research and needed tools. Failures of conveyor segments might cause congestion and deadlocks in the system. Hardly any literature has been found on this subject whether in semiconductor simulation studies or in analytical models. More attention should be paid to the relationship between control of the conveyor and the occurrence of failures to ensure a high reliability of the AMHS system.

Furthermore, hardly any attention has been paid to the relationship between conveyor systems and other material handling systems such as the stockers, more attention should be paid to synchronized scheduling of lot delivery and tools availability.

One of the advantages of conveyors is their ability to provide storage for lots, rather than sending them to stockers. Hence, research is needed to analyze the effect of recirculation on the overall behavior of the system.

With the exception of Rust et al. (2002), none of the studies addressing conveyor implementations for semiconductor fabs evaluate fab-wide key performance measures such the overall production, cycle time, or fab throughput, they mainly focused on AMHS-related measures, such as delivery times and AMHS throughput, a comprehensive evaluation is expected to investigate the effect on the overall fab performance measures such as cycle time, production tool utilization, and fab throughput (Montoya-Torres 2006).

Both analytical approaches and simulation can be used to solve such problems. Mathematical programming, queuing theory, and Markov decision processes can be used. Some of these techniques have proved to be successful in large vehicle-based systems. Most design and control problems in CFT systems require further research to:

- derive analytic methods to model large scales implementations of closed-loop conveyor material handling systems.

- develop analytical and simulation models which simultaneously address multiple design and control problems.

- develop analytical and simulation models which consider the joint optimization of design problems of conveyor systems and design problems of other material handling systems, such as stockers, under-track storage units, and hybrid vehicle-conveyor implementations.

- develop efficient analytical models for traffic management in large-scale conveyor systems with capacity constraints.

\section{CONCLUSION}

The tremendous development in semiconductor industry dictated the presence of highly automated systems. Mate- 
rial handling is a non-value adding process and should be effective and transparent. The existing automated material handling systems are vehicle-based systems. Continuous flow transport system (CFT) is considered an efficient alternative for car based systems as it eliminate the dependency on the car availability,

Research on semiconductor AMHS is entering a new phase with the increased usage of conveyor-based transport systems. From the literature survey we can conclude that most of the literature addresses application-specific simulation-based studies that are hard to generalize.

Research areas related to closed loop conveyors are identified, which include deriving analytic methods to model large scales implementations of closed-loop conveyor material handling systems, and developing analytical and simulation models which simultaneously address multiple design and control problems.

\section{REFERENCES}

Agrawal, G. K. and S. S. Heragu (2006). "A Survey of Automated Material Handling Systems in 300mm Semiconductor Fabs." IEEE TRANSACTIONS ON SEMICONDUCTOR MANUFACTURING 19(1).

Arzt, T. and F. Bulcke. (2000). A New Low Cost Approach in $200 \mathrm{~mm}$ and $300 \mathrm{~mm}$ AMHS. SEMICONDUCTOR FABTECH: 19-26.

Atmaca T. (1994). Approximate analysis of a conveyor system , Int.J Prod.Res, vol. 32, No.11, 26452655

Bastani. A. (1988). Analytical solution of closed-loop conveyor systems with discrete and deterministic material flow. ,European Journal of Operation Research, vol. 35, 187-192.

Bonora, T., M. Brain, and W. Fosnight, (2001). Reticle Automation Pathways for $300 \mathrm{Mm}$-Era Fabs. Yield Management Solutions: 49-52.

Bozer, Y., and Y-J Hsieh. (2004). Expected waiting times at loading stations in discrete-space closed loop conveyors. European Journal of Operation Research, vol. 155, 516-532

Brain, M., R. Gould, U. Kaempf, U., and B. Wehrung, (1999). Emerging Needs for continuous Flow FOUP Transport. International Electronics Manufacturing Technology Symposium.

Coffman, E. and E.Ngelenbe. (1988). Analysis of a conveyor queue in a flexible manufacturing system", European Journal of Operation Research vol. 35, 382-392.

Feindel, D. and U. Kaempf (2000). The Case for Continuous Flow Transport of Wafer Carriers. Semiconductor International.
Ferrell, J. and M. Pratt (2002). I300I Factory Guidelines: Version 5.0, International SEMATECH.

Foster, L. (2001). 300mm Wafer Factory Automation and the Logistics Infrastructure Challenge. FUTURE FAB. 11.

Garrett, T. (2001). Intel's 300mm Program Is on-Line, J. Electron. 2004.

Gartland, K. (1999). Automated Material Handling System (Amhs) Framework User Requirements Document: Version 1.0, International SEMATECH.

Heinrich, H. and A. Pyke. (1999). The Impact of Conveyor Transports on Factory Performance at Infineon's (Siemens) 200-mm Fab. SEMICONDUCTOR FABTECH: $15-18$.

Horn, G. W. and W. A. Podgorski (1998). A focus on cycle time-vs.-tool utilization "paradox' with material handling methodology. IEEE SEMI Advanced Semiconductor Manufacturing Conference.

Kuhl, M. E. and J. Christopher (2004). Capacity Analysis of Automated Material Handling Systems in Semiconductor Fabs. Proceedings of the 2004 Winter Simulation Conference.

Kuhl, M. E. and G. R. Laubisch (2004). A Simulation Study of Dispatching Rules and Rework Strategies in Semiconductor Manufacturing. IEEE/SEMI Advanced Semiconductor Manufacturing Conference.

Kwo T. (1958). A theory of conveyors. Management Science, Vol. 5, no. 1, 51-71.

Mayer, H. (1960). Introduction to conveyor theory. Western Electric Engineer vol.,4, no. 1, 43-47.

Montoya-Torres, J. R. (2006). "A literature survey on the design approaches and operational issues of automated wafer-transport systems for wafer fabs " Production Planning \& Control 17(7): 648-663.

Muth, E. (1972). Analysis of Closed-loop Conveyor Systems. IIE Transactions, vol. 4, no. 2, 134-143.

Muth E. (1975). Modeling and system analysis of multistation closed-loop conveyors, International Journal of Production Research, vol 13, No 6, 559-566

Muth E., and J. White. (1978). Conveyor Theory: A Survey", IIE Transactions, Vol11, No4, 270-277.

Paprotny, I., J.-Y. Shiau, Y. Huh, and G. Mackulak (2000). Simulation Based Comparison of semiconductor AMHS Alternatives: Continuous Flow vs. Overhead Monorail. Proceedings of the 2000 Winter Simulation Conference.

Roeder, T. M. and N. Govind (2004). A Queueing Network Approximation of Semiconductor Automated Material Handling Systems: How Much Information Do We Really Need?" Proceedings of the 2004 Winter Simulation Conference.

Schoeming, A. K. (1999). On the Corrupting Influence of Variability in Semiconductor Manufacturing. Proceedings of the 1999 Winter Simulation Conference, Phoenix, AZ. 
Shikalgar, S. T., D. Fronckowiak, and E. McNair (2002). $300 \mathrm{~mm}$ Wafer Fabrication Line Simulation Model. Proceedings of the 2002 Winter Simulation Conference.

Shon, B., D. Pillai, and Acker, N. "300 Mm Factory Design for Operational Effectiveness." Retrieved 27/3/2007, from http://www.intel.com/technology/silicon/ieee/30 00mmfactorydesign.pdf.

Singer, P. (2005). Is the Industry Ready for $450 \mathrm{~mm}$ Wafers? Semiconductor International.

Schmidt, L. and J. Jackman. (2000). Modeling Recalculating Conveyors with blocking. European Journal of Operations Research vol 124, 422-436.

Sonderman, D. (1982). An analytic model for recirculating conveyors with stochastic inputs and outputs, International Journal of Production Research, vol 20, No5, 591-605.

Tausch, F. and L. Hennessey (2002). Evaluation and Comparison of a Car-Based vs. a CFT Material Handling System for a 300mm Fab. Modeling and Analysis of Semiconductor Manufacturing Conference.

Therrien, R. J. (2002). The Power of Total Automation (Annual Report 2002), Brooks-PRI Automation.

Wright, R., M. Shopbell, K. Rust, and S. Sigireddy (2002). Effects of Metrology Load Port Buffering in Automated $300 \mathrm{~mm}$ Factories. Proceedings of the 2002 Winter Simulation Conference.

\section{AUTHOR BIOGRAPHIES}

DIMA NAZZAL earned her Ph.D. degree in industrial and system engineering from Georgia Institute of Technology, Atlanta, Georgia, in 2006. She is currently an Assistant Professor at the department of Industrial Engineering and Management Systems at the University of Central Florida, Orlando, Florida. Dr. Nazzal is a member of INOFRMS and IIE. Her research interests are in analytical modeling of manufacturing and logistics systems. She can be contacted by email at <dnazzal@mail.ucf.edu>.

AHMED EL-NASHAR.is a Ph.D. student in the Department of Industrial Engineering and Management Systems at the University of Central Florida. 